binary stars in star formation regions, satellite geodynamics, non-linear and nonstationary evolution of galaxies. Helioseismology studies carried out in the frame of the IRIS (International Research on the Interior of the Sun) and TON (Taiwan Oscillation Network) projects. Astrophysical programmes such as a search for periodicity in star-formation regions, study of close binary stars in the same regions, as well as in open clusters, CCD photometry of extra-galactic objects as gravitation lenses have been made at the Maidanak Observatory, which is located in the south-east of Uzbekistan. Monitoring of the seeing at Mt. Maidanak from 1996 to 1999, using ESO Differential Image Motion Monitor, showed that its atmospheric conditions are comparable with the best international observatories. The present status of the main fields of research and prospects are discussed. (Co-author is Shurat A. Ehgamberdiev, Uzbekistan.)

\title{
Astronomy in the Republic of Macedonia
}

Mijat Mijatovic, Institue of Physics, Skopje, Republic of Macedonia

Astronomy in the territory of today's Republic of Macedonia has a centurylong history. This history is presented in the essay, begining with M. Trpković's suggestions to reform the orthodox calendar in 1900s, through the foundation of the first faculty of Macedonian language in Skopje in 1946, until today's situation in astronomy.

In second half of the twentieth century, the development of astronomy in the Republic of Macedonia is divided in two different periods: before and after the big earthquake in Skopje in 1963. The first period is characterized by hope and enthusiasm, and a little observatory was started to be built, but it was destroyed in the earthquake. The last ten years a new upsurge is seen in Astronomy in the Republic of Macedonia, which is founded on Balkan and international collaboration.

\section{Astronomy without Astronomers?}

Magdalena Stavinschi, Astronomical Institute, Romanian Academy of Sciences, Cutitul de Argint 5, RO 75212 Bucharest, Romania

Astronomy in Romania has an old tradition. After half a century of privations and isolation from the rest of the world, we believed that the changes undergone by our country in 1989 (and by the neighbour countries, as well) will be benefit for the Romanian astronomy, too. Indeed, it was, but for a very short period. The young people left the country, one by one, and others cannot accept the low salary offered by a research institute. The economy doesn't allow us to enrich the astronomical endowment. Of course, we cannot close the observatories. We have to find other ways to save the astronomy in this part of Europe, especially in the epoch of the space astronomy. 